JURNAL

ANALYSE L'ACTE DE LANGAGE DIRECTIVE DE LA REQUETTE INDIRECTE DANS LES FILMS FRANÇAIS

\author{
Disusun dan Diajukan oleh: \\ Kiristina Lestaria Tarihoran
}

NIM : 2123331017

Telah Diverifikasi dan Dinyatakan Memenuhi Syarat untuk Diunggah pada Jurnal Online

Medan, Juli 2017

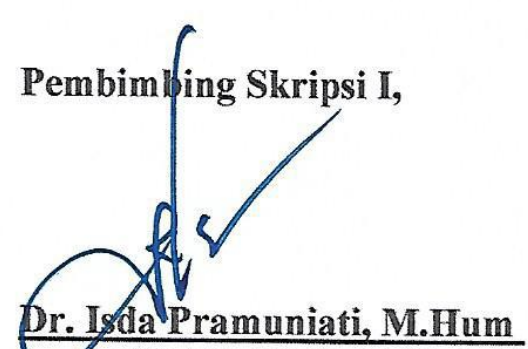

NFP. 196412071991032002
Menyetujui,

Pembimbing Skripsi II,

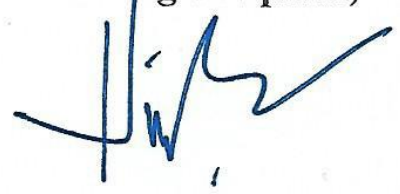

Dr. Junita Friska,M.Pd

NIP. 198006012005012003

Editor Jurnal

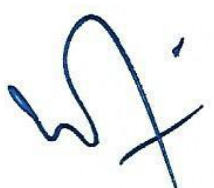

Nurilam Hariania, S.Pd., M.Hum

NIP. 197701212003122004 


\title{
ANALYSE L'ACTE DE LANGAGE DIRECTIVE DE LA REQUÊTE INDIRECTE DANS LES FILMS FRANÇAIS
}

\author{
Kiristina Lestaria Tarihoran \\ Dr. Isda Pramuniati, M.Hum \\ Dr. Junita Friska, M.Pd \\ Département des Langues Étrangères \\ Faculté des Lettres et des Arts \\ Université de Medan \\ Mel :hexagonefrance12@gmail.com
}

\begin{abstract}
RÉSUMÉ
Kiristina Lestaria Tarihoran, 2123331017, Analyse L'Acte de Langage Directive de la Requête- Indirecte Dans Les Films Français. Mémoire. Département des Langues Étrangère. Faculté des lettres et des Arts. Université de Médan. 2017.
\end{abstract}

Le but de recherche est de savoir les stratégies des actes de langage de la requêteindirecte de la Question sur Une Condition Réussite (QCR) trouvés dans les films français. La théorie dans cette recherche est la théorie de Warga .

La méthode utilisée est la méthode descriptive qualitative. Les donnés de cette recherche utilisent les cinq films français, c'est-à-dire Le Petit Prince, Jack et la mécanique du cœur, Ratatouille, Intouchables, et La Belle et La Bête.

Le résultat de cette recherche montre que la réussite de la demande à quelqu'un de faire quelquechose est montre de la condition et la stratégie. La réussite de la demande base de la stratégies est Question sur Une de Condition Réussite (QCR) en classifiedans cinq stratégies qui se trouvent dans les films français sont Question sur la capacité (30), Question sur la possibilité (1) qui utilise du verbe « pouvoir», Question sur la volonté (24) qui utilise du verbe «vouloir», Question sur la disponibilité ( 8) qui utilise du verbe «avoir», Question sur la permission (6) qui utilise du verbe «permettre». De ce résultat plus dominant utilise dans les films français est Question sur capacité parce qu'elle est plus facile utiliser dans tous les condition, elle a 30 ou $44 \%$, et la stratégie plus moins est la Question sur la possibilité (1) ou 1,5\% parce que elle utilise dans la possible situation.

On trouve quelques mode d'emploi pour exprimer la requête-indirecte QCR, ces sont le mode indicatif présent et conditionnel.

Mots clé : Acte de la requête Indirecte, QCR, Stratégie, Le Verbe et Le Mode, Les Films Français 


\begin{abstract}
ABSTRAK
Kiristina Lestaria Tarihoran, 2123331017. Analyse L'Acte Directive De Langage De La Requête Indirecte Dans Les Films Français. Skripsi. Program Studi Pendidikan Bahasa Prancis. Jurusan Bahasa Asing. Fakultas Bahasa Dan Seni. Universitas Negeri Medan. 2017.
\end{abstract}

Penelitian ini bertujuan untuk mengetahui strategi dari tindak tutur meminta secara tidak langsung dengan menggunakan pertanyaan berdasarkan keberhasilan sebuah kondisi dalam film- film Prancis. Teori yang digunakan adalah teori dari Warga .

Metode yang dipakai adalah metode deskriptif kualitatif. Sumber data. Sumber data penelitian menggunakan 5 film Prancis yaitu Le Petit Prince, Jack et La Mécanique du Cour, Ratatouille, Intouchables, et La Belle et La Bête.

Hasil penelitian ini menunjukkan bahwa keberhasilan dari sebuah tuturan permintaan ditunjukkan oleh kondisi dan strategi yang digunakan oleh penutur. Keberhasilan permintaan melalui strategi disebut dengan Question sur Une de Condition Réussite (QCR)terbagi atas lima strategi yang terdapat dalam film film Prancis yaitu Question sur la capacité (30) tuturan dengan verba pouvoir ,Question sur la possibilité (1) tuturan dengan menggunakan kata possible dan verba etre, Question sur la volonté (24) tuturan dengan menggunakan verba vouloir, Question sur la disponibilité (8) tuturan dengan verba yang digunakanadalahavoir, Question sur la permission (6)tuturan dengan verba permettre dan pouvoir. Dari penelitian ini strategi yang paling dominan ditemukan adalah Question sur la capacité dengan jumlah 30 tuturan karena strategi tersebut lebih mudah dipakai dalam setiap kondisi. Sedangkan tuturan dengan strategi Question sur la possibilité paling sedikit ditemukan dengan jumlah satu tuturan karena tergantung pada kemungkinan kondisi dari total 69 tuturan.

Dalam penelitian ini juga ditemukan penggunaan beberapa modus untuk meminta seseorang melakukan sesuatu yaitu Indicatif présent dan Conditionnel.

Kata Kunci : Tindak tutur meminta tidak langsung, QCR, Strategi, Verba dan Modus,Film - film Prancis

\title{
INTRODUCTION
}

La pragmatique est une étude du langage en acte ou bien l'accomplissement d'un certain nombre d'actes spécifiques. Kerbrat-Orecchioni (1995 : 22)considère que la parole est une forme d'action où les locuteurs transmettent des informations et de l'autre, ils tentent d'agir sur les interlocuteurs.Selon Austin (1962: 109), tous les énoncés sont performatifs, c'està-dire toute parole est une forme de l'action et ne pas simplement dire quelque chose.Jhon Langshaw Austin (1969) a classifié l'acte de langage en trois, ce sont : (1) L'acte locutoire qui exprime quelque chose, (2) l'acte Illocutoire qui est un 
acte pour faire quelque chose, et(3) l'acte perlocutoire qui est un acte effectué pour dire quelque chose.

Tandis que Searle a classifié acte illocutoire selon 5 catégories, ces sont (1) les actes assertives (les assertations constatives), (2) les actes directives (les actes de préscription: ordre, requête etc), (3) les actes permissives (consistant à s'éngager à faire quelque chose), (4) les actes expressifs (remercierement, felicitations, etc.), et (5) les actes déclaratives.L'acte de langage illocutoire directive est un acte de langage dans le quell'interlocuteur va faire quelque chose qui est demandé par le locuteur. Par exemple c'est l'acte de langage de la requête. La requête est classé comme un acte de langage directive car il permet aux participants de la communication d'interagrir bien afin que leur énoncée peut être comprise. L'acte de langage a classifié en deux ce sont acte de langage direct et acte de langage indirecte.Il peut définit que l'acte de langage de la requête indirecte est une acte qui demande à l'interlocuteur de faire quelquechose indirectement Selon Warga (2005: 147), l'acte de langage de la requête estdistingué trois types géneraux de formulations (directes, indirectes conventionnelles, indirectes non-conventionnelles).

Le type indirect conventionnelle dit que le destinataire comprend la valeur illocutoire de l'énoncé automatiquement meme si elle ne correspond pas à sa valeur locutoire.L'une de la stratégies de l'acte de langage de la requête inderecte conventionnelle utilise QCR (Questions sur Une Condition de Réussite).Il existe cinq strategies différentes qui reposent toutes sur une des conditions de réussite de la requête: (1) Question sur la capacité, (2) Question sur la possibilité, (3) Question sur la volonté, (4) Question sur la disponibilité, (5) Question sur la permission.

Question sur la capacité Pour questionner sur la capacité de l'auditeur (interlocuteur), on emploie souvent le verbe " pouvoir » au présent de l'indicatif ou au conditionnel présent. Est-ce que vous pourriez m'aider? Question sur la possibilitéEst-il possible d'emmener le chien a l'ecole?Locuteur demande à l'interlocuteur de faire quelque chose selon la possibité en utilisant une forme interogatif (QCR). Ces sont remarquée par l'usage du mot «possible» et du verbe «être» au present ou conditionnell. Question sur la volonté Question sur la volonté de l'auditeur à accomplir l'actepar exemple Voudrais-tu m'aider? Locuteur demande à l'interlocuteur de faire quelque chose selon la volonté (intention) en utilisant une forme interogatif (QCR). Ces sont remarquée par l'usage du mot «vouloir » au conditionelle. Question sur la Disponibilité (Avezvous le temps de m'aider?) Locuteur demande à l'interlocuteur de faire quelque chose selon la disponibilité en utilisant une forme interogatif (QCR). Ces sont remarquée par l'usage du mot «avoir » au présent et conditionelle. Question sur la permission Locuteur demande à l'interlocuteur de faire quelque chose selon la permission en utilisant une forme interogatif (QCR). Ces sont remarquée par l'usage du mot " pouvoir » au conditionelle. Par exemple Puis-je prendre mon chien a l'ecole?. Il est important que la stratégie indirecte sur QCR soit bien attentionné et analysé car l'existence de ce stratégie n'est pas bien exposée dans 
l'apprentissage et la conversation de quotidienne. Les étudiants ne savent pas que les strategies de l'acte de langage de la requête indirecte peut indiquer le mode dans le indicatif et conditionnel pour savoir la polithèsse. À cause de la connaissance limité, il est possible qu'un apprenant à de la difficulté à savoir et utiliser l'acte de la requête indirecte sur QCR dans unconversation plus large que le texte.

Par consequent, cette recherche est très importante à faire pour informer le lecteur, en particulier les apprenants de français sur l'acte de langage directive de la requête indirecte afin qu'ils puissant comprendre la stratégie le locuteur demande à quelqu'un de faire quelque chose à l'écrit ou à l'oral.

\section{MÉTHODOLOGIE DE LA RECHERCHE}

La méthode de recherche est très importante dans une recherche afin que le résultat obtenu soit satisfaisant. Avec la méthode de recherche correcte, les problèmes apparus dans la recherche peuvent être résous et le chercheur peut trouver les vérités, si bien que dans l'analyse de données, il peut être bien dirigé. Le processus de recherche qui est orientée va produire l'information et l'analyse de données.

Selon Sujoko, Stevanus, Yuliati (2007 : 7) déclare que metode penelitian merupakan bagian dari metodologi yang secara khusus mendeskripsikan tentang cara mengumpulkan data dan menganalisis data. Ca veut dire, la méthode de la recherche est une partie de méthodologie, qui est en particulièrement exprimer la manière de collecter et analyser des données. Ainsi, on peut signifier la méthode de la recherche est une façon scientifique de gagner les données systématiquement. Donc, dans cette recherche on utilise la méthode descriptive qualitative.

Selon Bogdan et Taylor Margono (2005 : 36) dit que, penelitian kualitatif ialah proses penelitian yang menghasilkan data deskriptif berupa kata-kata tertulis atau lisan dari orang-orang yang diamati. Ca veut dire, est un procès de la recherche qui produit la donnée descriptive comme le mot à l'écrit ou à l'oral de personnes observées.

Les procédures d'analyse de données dans cette recherche se composent de :

1. Chercher les références dans lesquels il y a des explications sur acte de langage de la requête indirecte QCR.

2. Lire et comprendre les explications sur l'acte de langage de la requête indirecte.

3. Regarder bien les films français en trouvant la transcription du film.

4. Trouver les actes des langages de la requête et les stratégies indirecte dans le film.

5. Grouper les stratégies de l'acte de langage de la requête indirect QCR selon les stratégies.

6. Analyser les verbes et les modesde l'acte de langage de la requêteindirect QCR dans les films. 
7. Faire une conclusion en donnant les suggestions possibles.

\section{RÉSULTAT DE LA RECHERCHE}

\section{a. Question sur la capacité}

1. La police à sa maman de petit fille: Vous pourriez lui parler?(Le Petit Prince)(00:45:21)

Un énoncé au -dessus sont la requête- indirecte. Locuteur (La police) demande à l'interlocuteur (maman) de faire quelque chose dans cette situation la police demande à maman de la petite fille parler à sa petite fille. selon la capacité en utilisant une forme interogatif (QCR). C'est remarquée par l'usage du verbe « pouvoir» au conditionelle car il fait posé comme l'hypothèsse et exprimer une demande polie.

\section{b. Question sur la possibilité}

\section{2.la petite fille au renard :C'est possible? Ratatouille (01:46:07)}

Un énoncé au -dessus sont la requête- indirecte. Locuteur (la petite fille) demande à l'interlocuteur (renard) de faire quelque chose. dans cette situation la petite fille demander au renard rendre la carte identité de Monsieur prince . selon la possibilité en utilisant une forme interogatif (QCR). La question sur possibilité est remarquée par l'usage du mot «possible».

\section{c. Question sur la volonté}

3. Gusteau à Rémy :Est-que vous voulez de bouchées dynamyques?(Ratatouille) (1:12:00)

Unénoncé au -dessus est la requête indirecte. Locuteur demander a l'interlocuteur de faire quelque chose selon la volonté en utilisant une forme interogatif (QCR), c'est -à- dire du mot est-ce que. La parole audessus est remarquée par l'usage du verbe «pouvoir » dans le mode «présent» et utilise le pronom personnelle «vous» qui indique la situation est formelle ( locuteur et interlocuteur ne se conaissent pas).

\section{d. Question sur la disponibilité}

Dans cette recherche la stratégie question sur la disponibilité qui indiquer la locuteur demande à l'interlocuteur.

4.La rose au Petit Prince : Auriez- vous la bonte penser de moi?(Le Petit Prince)(29:10)

Un énoncé au -dessus est la requête- indirecte. Locuteur demande à l'interlocuteur de faire quelque chose selon la disponibilité en utilisant une 
forme interogatif (QCR). Ce sont remarquée par l'usage du verbe « avoir» au « conditionnelle» un énoncé ci- dessous est plus polie parce que locuteur utilise le mode conditionnel qui exprimer une demander polie.

\section{e. Question sur la permission.}

5. Remy à Gusteau : C'estvotre testament. Oh c'est interressant. Permettez que je....?Ratatouille (01:22:00)

Un énoncée au -dessus sont la requête- indirecte. Locuteur demande à l'interlocuteur de faire quelque chose selon la permission en utilisant une forme interogatif. C'est remarquée par l'usage du verbe «permettre» au conditionnelle. Les énoncées ci-dessus a la même situations, c'est - à - dire dans un bureau.

\section{CONCLUSION}

Les strategies de l'acte de la requête-indirecte sur QCR se composent cinq strategies différents qui reposent toutes sur une des Conditions Réussite de la requête suivant: Question sur la capacité, question sur la possibilité, Question sur la volonté, question sur la disponibilité, question sur la permission. Le stratégies de la requête -indirecte QCR le plus fréquent d'utilisation est Question sur la capacité, c'est 33. Tandis que les stratégies question sur la possibilité le moins fréquent d'utilisation, c'est un. Et on peut facilement observer que question sur la capacité au mode conditionnel occupe la place la plus importante dans ces films français. La question sur la capacité est comme le sujet ou le premier stratégies dans les films français. C'est à dire que ces films en parle.

\section{BIBLIOGRAPHIE}

Ali, KHERBACHE. 2000. Quel Rapport a La Culture Parlée-Ecrite : Université de Souk-Ahras, Algérie.

Austin J L. 1962. How To Do Things With Words. 2ème edition. Clarendon Press : Oxford

Avodo, Joseph. 2012. La politesse linguistique dans la relation interlocutive en classe : des enjeux de faces aux enjeux opératoires : University of Bergen.

Brown, D. \& S. Levinson. 1987. Politeness. Cambridge University Press : London

Crystal, D. 2010. The Cambridge Encyclopedia of Language. 3ème edition. Cambridge University Press : Cambridge.

Fallou, MBOW. 2011. L'Acte de Requête Dans L'Interaction Verbale : Université Cheikh Anta DIOP, Sénégal. 
Grandcolas, Berbadette. 2000. Comment peut-on enseigner la politesse en langue étrangère? Journal des langues modernes

Holttinen, Tuuli. 2013. Les requêtes chez les apprenants de FLE : Université de Helsinki

KerbratOrechioni C. 2001. Les Interactions Verbales. Armand Collin : Paris

Kherbache, Ali. 2000. Quel Rapport à la Culture Parlée-Ecrite ?.Departement de français, Université de Soule-Ahras. Algérie.

Leech, G.N. 1983. Principales of Pragmatics.Longmann : Londres et New York

Levinson, Stephen C. 1983. Pragmatics : Cambridge University Press, London.

Levinson, Stephen. C. 2000. Journal of Linguistics Antrophologhy

Meier, A.J. 1997. Passage of Politeness. Journal of Pragmatics

Moescler, J. \& A. Reboul 1994. Dictionnaire Encyclopédique de Pragmatique. Seuil : Paris

Nadar, F.X. 2009. Pragmatik dan PenelitianPragmatik: Grahallmu, Jakarta.

Reboul, Anne, dkk. 1998. Pragmatique du Discours :De l'interprétation de l'énoncé à l'interprétation du discours : Armand Collin, French.

Searle, John R. 1970. Speech Acts : An Essay in the Philosophy of Language:Cambridge University Press, Viet, Do Quang. 2010. Regards Croisés sur l'Acte de Requête : Université National de Hanoi.

Searle, J.R. 1972. Les Actes de Langage.Hermann : Paris

Searle J R. 1975. Indirects Speechs Acts. Academic Press : New York.

Sundari, Dewi.2015. Analyse des Erreurs Grammaticales de L'acte de Langage de Refus des Etudiants du Quatrième Semestre.

Villemin, F-Y. 2012. Les Actes de Langage. Conservatoire National Disaris Métiers.

Viljamaa, Heidi. 2012. Emploi des requêtes et des remerciements chez des étudiants finlandais : Université de Turku : London.

Walija. 1996. BahasaIndonesiaDalamPerbincangan: IKIP Muhammadiyah Jakarta Press, Jakarta.

Warga. M. 2007. Interlangue Pragmatic in L2 French. John Benjamin: Amsterdam et Philadephia. 
Wibowo, Wahyu. 2001. Pengorganisasian Karangan Pragmatik dalam Bahasa Indonesia untuk Mahasiswa dan Praktisi Bisnis : Gramedia Pustaka Utama, Jakarta.

\section{SITOGRAPHIE}

https://www.allocine.fr/film/fichefilm_gen_cfilm=182745.htmlAccèsau25 février2017)

http ://www.wikipedia.org/wiki/Le_Petit_Prince_(film,_2015)Acces au 25 février2017)

http//fr.m.wikipedia.org/wiki/Jack_et_la_Méchanique du cœur. Acces au 25février2017)

http:// fr.m.wikipédia.org/wiki/Intouchables_(film)Acces au 25 février2017. http :fr.m.wikipédia.org/wiki/Ratatouille_(film) Acces au 25 février2017. 